\section{(2) OPEN ACCESS}

\title{
Antigen-driven selection of antibodies against SSA, SSB and the centromere 'complex', including a novel antigen, MIS12 complex, in human salivary glands
}

\author{
Masaru Takeshita (D) ,' Katsuya Suzuki, ${ }^{1}$ Yukari Kaneda, ${ }^{1}$ Humitsugu Yamane, \\ Kazuhiro Ikeura, ${ }^{2}$ Hidekazu Sato, ${ }^{2}$ Shin Kato, ${ }^{2}$ Kazuyuki Tsunoda, ${ }^{2}$ Hisashi Arase, ${ }^{3,4}$ \\ Tsutomu Takeuchi ${ }^{1}$
}

\begin{abstract}
Handling editor Josef S
Smolen

- Additional material is published online only. To view please visit the journal online(http://dx.doi.org/10.

1136annrheumdis-2019215862)
\end{abstract}

${ }^{1}$ Division of Rheumatology, Department of Internal

Medicine, Keio University School of Medicine, Shinjuku-ku, Japan

${ }^{2}$ Division of Oral and

Maxillofacial Surgery,

Department of Dentistry and

Oral Surgery, Keio University

School of Medicine, Shinjuku-ku,

Japan

${ }^{3}$ Department of

Immunochemistry, Research Institute for Microbial Diseases

Osaka University, Suita, Japan

${ }^{4}$ Laboratory of

Immunochemistry, WPI

Immunology Frontier Research

Center, Osaka University, Suita, Japan

\section{Correspondence to}

Professor Tsutomu Takeuchi, Division of Rheumatology, Department of Internal Medicine, Keio University School of Medicine, Shinjuku-ku, Tokyo, Japan; tsutake@z5.keio.jp

Received 13 June 2019 Revised 24 August 2019 Accepted 14 September 2019 Published Online First 14 October 2019

\section{Check for updates}

(C) Author(s) (or their employer(s)) 2020. Re-use permitted under CC BY-NC. No commercial re-use. See rights and permissions. Published by BMJ.

To cite: Takeshita $\mathrm{M}$

Suzuki K, Kaneda Y,

et al. Ann Rheum Dis

2020;79:150-158

\section{ABSTRACT}

Objectives Recent evidences have revealed that anti-SSA/SSB antibodies, the major autoantibodies in Sjögren's syndrome (SS), are produced in salivary glands. This study aims to clarify overall of autoantibody production at lesion site, including anti-centromere antibody (ACA)-positive SS.

Methods Antibodies of antibody-secreting cells in human salivary glands were produced as recombinant antibodies. The reactivity of these antibodies and their revertants were investigated by ELISA and newly developed antigen-binding beads assay, which can detect conformational epitopes. The target of uncharacterised antibodies was identified by immunoprecipitation and mass spectrometry. Autoantibody-secreting cells in salivary gland tissue were identified by immunohistochemistry using green fluorescent protein-autoantigen fusion proteins.

Results A total of 256 lesion antibodies were generated, and 69 autoantibodies including 24 ACAs were identified among them. Beads assay could detect more autoantibodies than ELISA, suggesting autoantibodies target to antigens with native conformation. After somatic hypermutations were reverted, autoantibodies drastically decreased antigen reactivity. We showed that MIS12 complex, a novel target of ACA, and CENP-C are major targets of ACA produced in salivary glands by examining cloned antibodies and immunohistochemistry, whereas few antiCENP-B antibodies were detected. The target profiling of serum ACA from 269 patients with SS, systemic sclerosis $(\mathrm{SSC})$, primary biliary cirrhosis $(\mathrm{PBC})$ and healthy controls revealed that $A C A$-positive patients have antibodies against various sites of centromere complex regardless of disease.

Conclusion We showed direct evidences of antigendriven maturation of anti-SSA/SSB antibody and ACA in SS lesion. ACA recognises centromere 'complex' rather than individual protein, and this feature is common among patients with SS, SSC and PBC.

\section{INTRODUCTION}

Serum autoantibody tests are widely used in clinical practice because the type of autoantibody is related to clinical course, treatment response and prognosis. ${ }^{12}$ Sjögren's syndrome (SS) is an autoimmune disease that is characterised by chronic lymphocyte infiltration into salivary and lacrimal glands, that

\section{Key messages}

What is already known about this subject?

- The typical autoantibody of Sjögren's syndrome (SS), anti-SSA and anti-SSB antibody, is produced in salivary glands.

- Anti-centromere antibody (ACA) is the major autoantibody detected in limited-cutaneous systemic sclerosis and primary biliary cirrhosis (PBC) but it is also detected in a part of SS patients' serum.

What does this study add?

- In addition to anti-SSA/SSB antibody, ACA is also produced in the salivary glands of SS patients in an antigen-driven manner.

- SerumACA of patients with SS, SSC and PBC are commonly targeted to various sites of the centromere 'complex', not to individual proteins.

How might this impact on clinical practice or future developments?

- This study highlights the significance of detail immunological analysis in local lesion for better understanding of disease-relevant autoantibody and classification of autoimmune diseases.

results in destruction of glands and causes sicca syndrome. ${ }^{3}$ Anti-SSA antibody, which is collective term for multiple antibodies reacting to the SSA52 (TRIM21) and SSA60 (TROVE2), and anti-SSB antibody are the most commonly detected autoantibody in SS, and actually, anti-SSA antibody are used as one of the classification criteria of SS. ${ }^{4}$

Other autoantibodies, including anti-centromere antibody (ACA), anti- $\alpha$-fodrin antibody and anti-muscarinic receptor three antibody, are also reported in a part of SS patients. ${ }^{5} \mathrm{ACA}$ is the major autoantibody detected in limited-cutaneous systemic sclerosis $(\mathrm{lcSSc})^{6}$ and primary biliary cirrhosis (PBC), ${ }^{7}$ and clinically used in the classification criteria for systemic sclerosis $(\mathrm{SSc}),{ }^{8}$ but not included in the classification criteria for SS. In practice, ACA is detected by anti-nuclear antibody (ANA) test as discrete-speckled pattern (corresponding to the centromere region of chromosome). ELISA of anti-CENP-B antibody is also used because this result is highly consistent with ACA by 
ANA test. ${ }^{9}$ In addition, CBX5, CENP-A, CENP-B and CENP-C are known to be the targets of ACA, although the reactivity against these proteins was reported to vary slightly depending on the disease; antibody against CBX5 and N-terminus of CENP-C are appear more frequently in SS than in $\mathrm{SSc}^{10}$; patients with primary SS have antibody that predominantly recognise CENP-C alone, whereas patients with SSc have antibody that recognise both CENP-B and CENP-C ${ }^{11}$.

In addition to autoantibodies, there are similarities in clinical symptoms among ACA-positive diseases. ACA-positive SS has higher prevalence of Raynoud's phenomenon and sclerodactyly, which are the major symptoms of ACA-positive SSc, than SS without ACA. ${ }^{12} 13$ However, the mutual relationship between these ACA-positive diseases has not been clarified.

Accumulating experimental evidence has identified the characteristics of anti-SSA/SSB antibody in SS as follows: IgA-type anti-SSA/SSB antibody are secreted into saliva ${ }^{14}$; anti-SSA/SSB antibody-secreting cells (ASCs) exist in salivary glands ${ }^{15}{ }^{16}$; and $\mathrm{B}$ cells proliferate clonally in the salivary glands in an antigendriven manner. ${ }^{17-19}$ Recently, advanced single-cell technology has enabled the analysis of immunoglobulin heavy chain $(\operatorname{IgH})$ and light chain $(\mathrm{IgL})$ of each individual cell; therefore, we can reproduce antibody produced in vivo as recombinant proteins in vitro. ${ }^{20}$ Several groups have reported that a high frequency of autoreactive/polyreactive antibody is observed in CD21-/low and naïve B cells in peripheral blood of patients with SS, ${ }^{21-23}$ whereas others reported increased frequencies of IgG-type ASCs in salivary glands that react to multiple autoantigens simultaneously. ${ }^{24}$

These studies have gradually revealed the nature of autoantibody produced in lesion site of SS. Therefore, we aim to clarify the overview of the autoantibody production at the lesion site including ACA-positive SS. Here, we isolated ASCs from salivary glands of patients with SS and comprehensively and efficiently reproduced their antibodies. We called these antibodies, produced by lymphocytes infiltrating in the disease lesion site, as 'lesion antibody', and by examining the reactivity of our lesion antibody panel, we revealed antigen-driven production of autoantibody against SSA, SSB and centromere 'complex' including a novel centromere-related autoantigen. Finally, we profiled the targets of ACA in patients with SS, SSc and PBC, and clarified the common reactivity of autoantibody among these patients.

\section{METHODS}

Detailed methods are described in online supplementary file 1. We created 256 recombinant antibodies of ASCs in salivary glands from nine patients. The reactivity of these antibodies were investigated by ELISA and a newly developed procedure, antigen-binding beads assay. Antibodies against CBX5, CENP-A, CENP-B, CENP-C and MIS12 complex (MIS12C) were examined in 269 serum and 11 salivary gland tissues.

\section{RESULTS \\ Identification of anti-SSA/SSB antibody in lesion antibody panel}

The IgH and IgL variable regions of ASCs derived from salivary glands were sequenced and used to produce lesion antibody panel. Patients' background and the number of antibodies are shown in online supplementary table 3 . Of the 352 cells sorted, 256 recombinant antibodies were produced, the overall success rate was approximately $73 \%$.

We first checked the polyreactivity of them since antibody derived from patients with autoimmune diseases are known to include polyreactive antibody. ${ }^{25}$ The reactivity to lipopolysaccharide (LPS), insulin and dsDNA were examined, and antibody that reacted to two or more antigens were considered polyreactive. Among 256 antibodies, only three antibodies, LB20-34, LB20-80 and LB24-9, were polyreactive.

Next, we examined the reactivity of lesion antibodies using ELISA for anti-SSA52, anti-SSA60 and anti-SSB antibody. In addition, we examined the reactivity to antigens with native conformation by antigen-binding beads assay. Because SSA52 has Fc receptor activity, ${ }^{26}$ all IgGs bound to SSA52-binding beads. Therefore, we used point mutated SSA52 (D355A), which lost Fc receptor activity, ${ }^{27}$ to examine the reactivity against SSA52. Antibodies that exhibited reactivity by either ELISA or beads assay were regarded as positive. Antibodies binding to more than one antigen was counted as antibody for antigen with the highest titre. Since two polyreactive antibody, LB20-34 and LB20-80, bound to all three antigens but had the highest titre against SSB, they were counted as anti-SSB antibody.

Anti-SSA/SSB antibody profiles of serum and corresponding lesion antibodies are shown in online supplementary table 4. We identified seven anti-SSA52 antibodies, 23 anti-SSA60 antibodies and 15 anti-SSB antibodies in our panel. As previously reported, ${ }^{15}$ autoantibodies were cloned only from the patients who were serum autoantibody positive. Between two detection procedures, antigen-binding beads assay had more sensitivity. There were six anti-SSA52, 15 anti-SSA60 and seven anti-SSB antibodies that were negative by ELISA but positive in beads assay. Among ASCs in salivary glands from serum anti-SSA/ SSB antibody-positive patients, $30.6 \%$ of ASCs could produce anti-SSA/SSB antibody.

\section{Antigen-driven selection of anti-SSA/SSB antibody}

Next, we focused on 12 anti-SSB antibodies found in one patient (LB20), and examined whether the epitopes of these antibody were identical. Since multiple epitopes of serum anti-SSB antibodies have been identified by western blotting, ${ }^{528}$ we expressed three truncated forms of SSB protein, 1-107 amino acids (AA), 108-242 AA, and 243-408 AA, and examined which form the antibody reacted to. As shown in figure 1A, epitopes differed depending on antibodies, suggesting that they were selected against whole SSB protein. Although LB20-34 and LB20-80 used in this experiment were polyreactive antibody, the epitope of SSB exists only in 1-108 AA region, indicating that the epitope recognised in one molecule could be specific, even among polyreactive antibody.

To directly examine whether the obtained autoantibody was selected in an antigen-driven manner, we created 17 revertant antibodies, which have all somatic hypermutations (SHMs) reverted to the genomic sequence, and examined the reactivity using ELISA and antigen-binding beads assay (figure 1B-C). All of the revertant antibodies showed drastically decreased antigen reactivity by both ELISA and beads assay. Notably, two revertant antibodies created from anti-SSB antibody with polyreactivity lost polyreactivity after SHMs were reverted. Our results directly proved that most preselected autoantibody have no or poor binding ability, but they are selected and refined against autoantigens by accumulating SHMs.

\section{Identification of a novel autoantigen in ACA-positive SS patient}

In our cohort, one patient (LB32) was serum ACA-positive by ANA test and serum anti-CENP-B antibody-positive by ELISA. We next examined whether ACA, like anti-SSA/SSB antibody, are also produced in salivary glands. First, we checked whether 
A
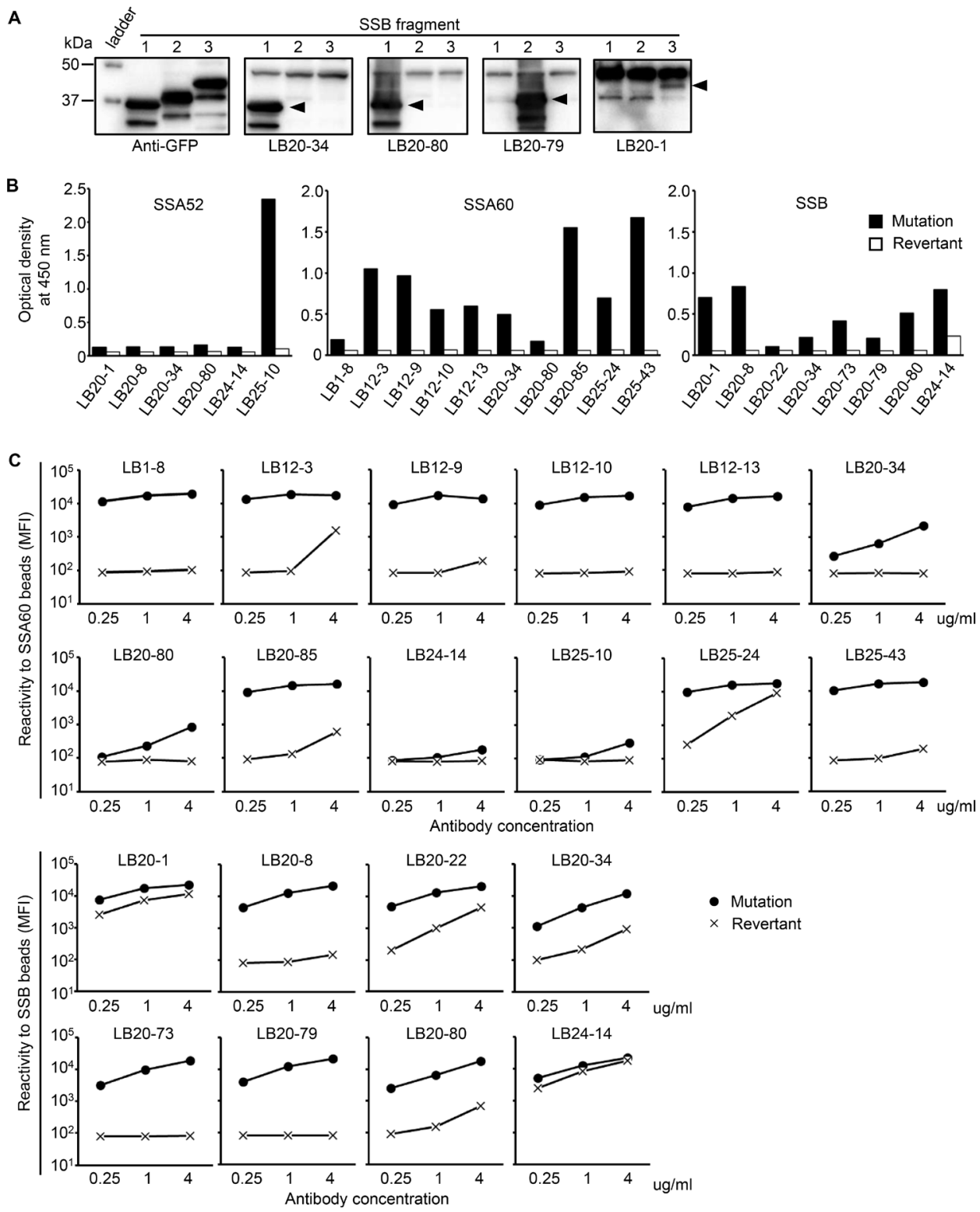

Figure 1 Direct evidence of antigen-driven antibody selection against SSA and SSB. (A) GFP-fusion SSB fragments (Lane 1: 1-107 AA, Lane 2: 108-242 AA, and Lane 3: 243-408 AA) were isolated by SDS-PAGE. Anti-SSB antibody from one patient (LB20) was examined to determine which fragment they bound to. Anti-GFP antibody was used as a positive control. The band common to all anti-SSB antibodies at approximately $47 \mathrm{kDa}$ was considered endogenous SSB. (B) Anti-SSA52, anti-SSA60 and anti-SSB antibody and their revertants were tested by ELISA at a concentration of $2 \mu \mathrm{g} /$ $\mathrm{mL}$. The vertical axis shows the OD at $450 \mathrm{~nm}$. (C) Anti-SSA60 and anti-SSB antibody and their revertants were tested in antigen-binding beads assay. The reactivity of the antibody bound to SSA60-binding and SSB-binding beads was measured by flow cytometry. AA, amino acid; OD, optical density; SS, Sjögren's syndrome.

antibodies from LB32 react to CENP-B by ELISA, and surprisingly, none of the 39 antibodies reacted to CENP-B. For confirmation, we tested another manufacturer's ELISA, but the results did not change.

To investigate what these antibodies react to, we performed immunoprecipitation with a K562 cell lysate using these antibodies. Several proteins were precipitated by one of these antibodies, and identified by mass spectrometry (figure $2 \mathrm{~A}$, online supplementary file 2). This result was verified by western blotting using antibody to DSN1, MIS12 and MIS12C (figure 2B), and we further revealed that several antibodies derived from LB32 had similar reactivity.

These precipitated proteins were known to be constituents of KMN assembly in kinetochore complex. ${ }^{29} \mathrm{KMN}$ assembly consists of three subcomplexes, KNL1 complex (KNL1C),
MIS12C and NDC80 complex (NDC80C), and the constituent proteins of these subcomplexes are shown in figure $2 \mathrm{C}$. We speculated that these antibodies precipitated whole KMN assembly; therefore, we next examined which protein the antibodies bound to. We expressed each protein in $293 \mathrm{~T}$ cells, and used them for antigen-binding beads assay. As shown in figure $2 \mathrm{D}$, antibodies derived from LB32 did not bind to ZWINT1, a constituent of KNL1C, or to NUF2, SPC24 or SPC25, constituents of NDC80C, whereas they weakly bound to DSN1, NSL1, MIS12 and PMF1, constituents of MIS12C. Because it is unlikely that one monoclonal antibody $(\mathrm{mAb})$ binds to four individual proteins, we speculated that when one of MIS12C constituent proteins was expressed, it would form complex together with other endogenous constituents, and antibody recognised the conformation of MIS12C. To verify this, we cotransfected all four constituent 
A

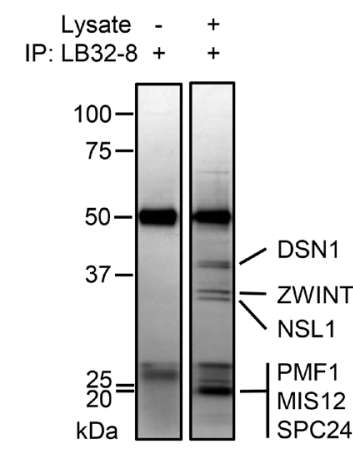

B

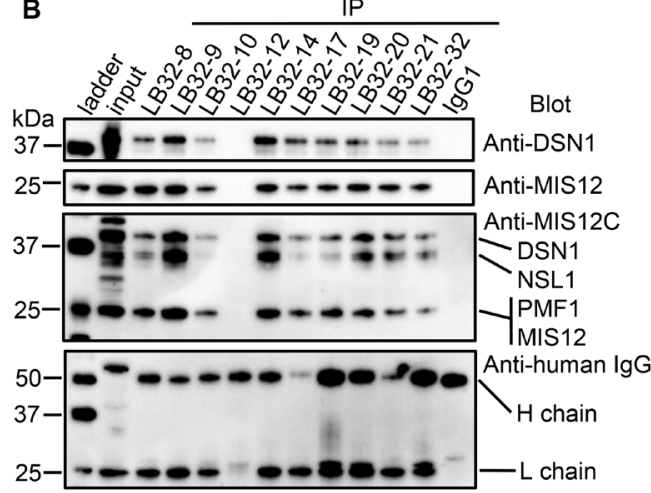

C

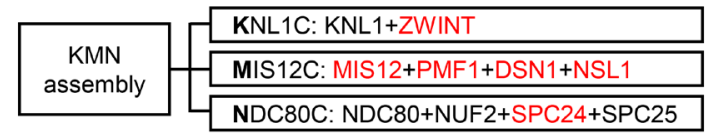

D
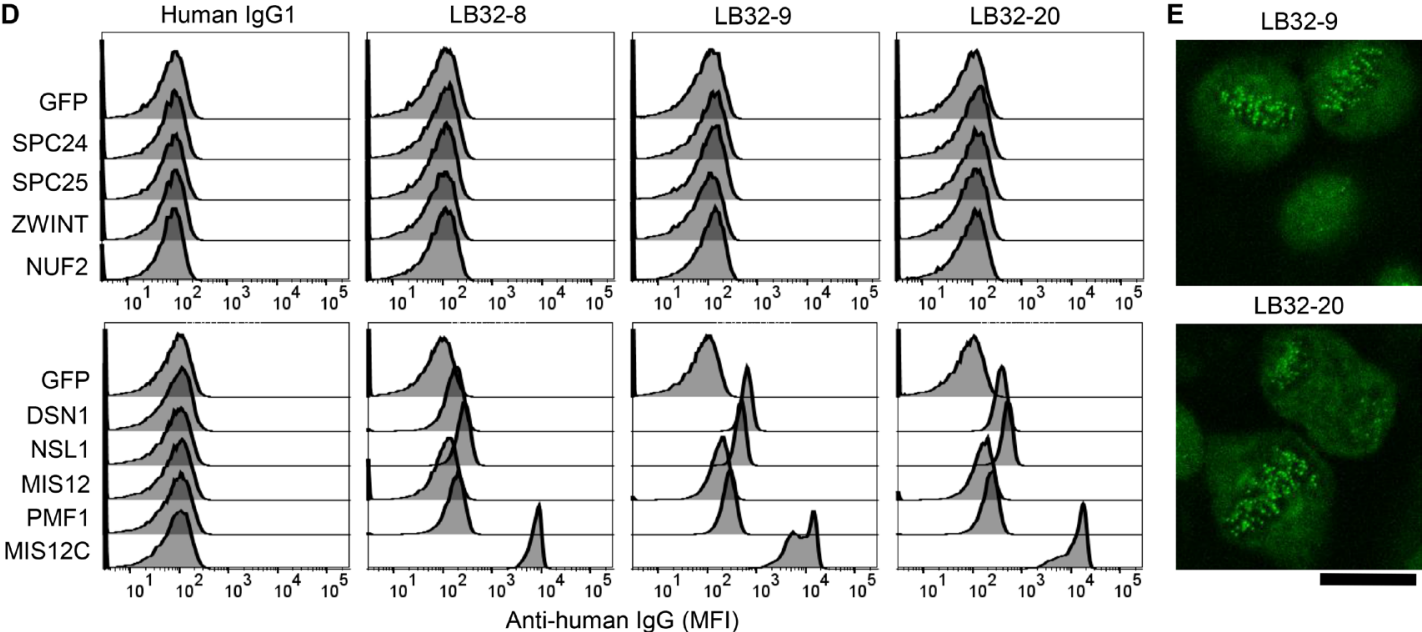

LB32-20

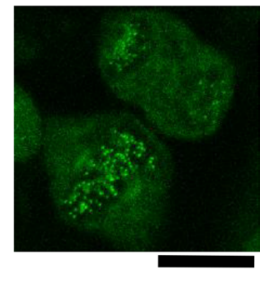

Figure 2 Identification of the target of the lesion antibody derived from serum ACA-positive patients. (A) Immunoprecipitation was performed with cell lysate of K562 cells or lysis buffer using the LB32-8 antibody. The precipitated proteins were electrophoresed and stained by silver staining. The names of proteins identified by mass spectrometry are shown. The common bands at $50 \mathrm{kDa}$ and $25 \mathrm{kDa}$ are the heavy and light chains of the antibody, respectively. (B) The proteins that were immunoprecipitated from K562 cell lysate by each LB32 antibody were electrophoresed, and blotted with antibody against DSN1, Mis12 complex (MIS12C) and human IgG. (C) Three subcomplexes constituting the KMN assembly and their constituent proteins are shown. The identified proteins by mass spectrometry are shown in red. (D) The reactivity of antibody from LB32 was examined by antigen-binding beads assay. The indicated proteins were coupled with streptavidin beads. The reactivity against each autoantigen-binding beads was measured by flow cytometry. (E) An indirect fluorescent antibody test (ANA) of HEp-2 cells was performed using the anti-MIS12C antibody, LB32-9 and LB32-20, at a concentration of $50 \mu \mathrm{g} / \mathrm{mL}$. Scale bar indicates $20 \mu \mathrm{m}$. ACA, anti-centromere antibody; ANA, anti-nuclear antibody.

proteins of MIS12C and found that the reactivity was remarkably increased (figure 2D), suggesting that these antibodies react to the complex form of MIS12C.

We further examined anti-MIS12C antibodies by ANA test. As expected, anti-MIS12C antibody showed discrete-speckled patterns (figure 2E). Our results revealed that anti-MIS12C antibody is a novel member of ACA.

\section{Identification of autoantibodies against various centromere- related proteins}

Next, we examined the reactivity of all of our lesion antibodies against MIS12C. In addition, other known targets of ACA, including CBX5, CENP-A, CENP-B and CENP-C, all of which are known to bind to MIS12C, ${ }^{29-31}$ were also examined.

As shown in online supplementary table 5 , antibodies derived from LB32 contained many anti-MIS12C and anti-CENP-C antibodies, and interestingly, antibodies derived from LB02, a serum ACA-negative patient, contained multiple types of ACA.
Considering the time course between autoantibody production in lesion and its detection in serum, we checked the serum of LB02 2 years after salivary gland biopsy; however, it was still ACA negative. This finding indicates that the serum and salivary gland autoantibody profiles are not always consistent. In these two patients, approximately one-third of the cloned antibodies was ACA, and this proportion was comparable to that of anti-SSA/SSB antibody. Although one anti-CBX5 antibody was found among antibodies from LB01 (serum ACA negative, serum anti-CENP-B antibody negative), the possibility of a coincidence occurring could not be denied. A total of 24 ACA were found in our lesion antibodies by beads assay, but curiously, anti-CENP-B antibody could not be found in salivary glands, as previously shown by ELISA.

\section{Antigen-driven selection of ACA}

We next investigated whether ACA, like anti-SSA/SSB antibody, was selected in an antigen-driven manner. MIS12C is known to 
A

LB2-18

LB32-8

LB32-9

LB32-14

LB32-17

LB32-32
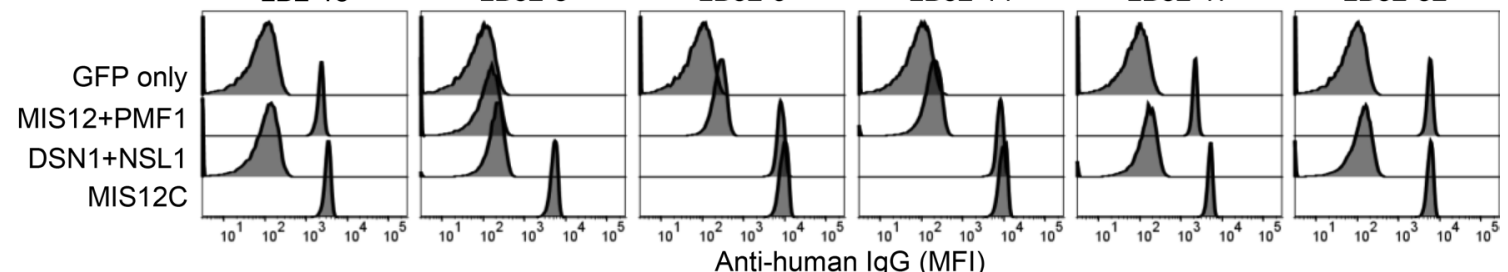

Anti-human IgG (MFI)

B

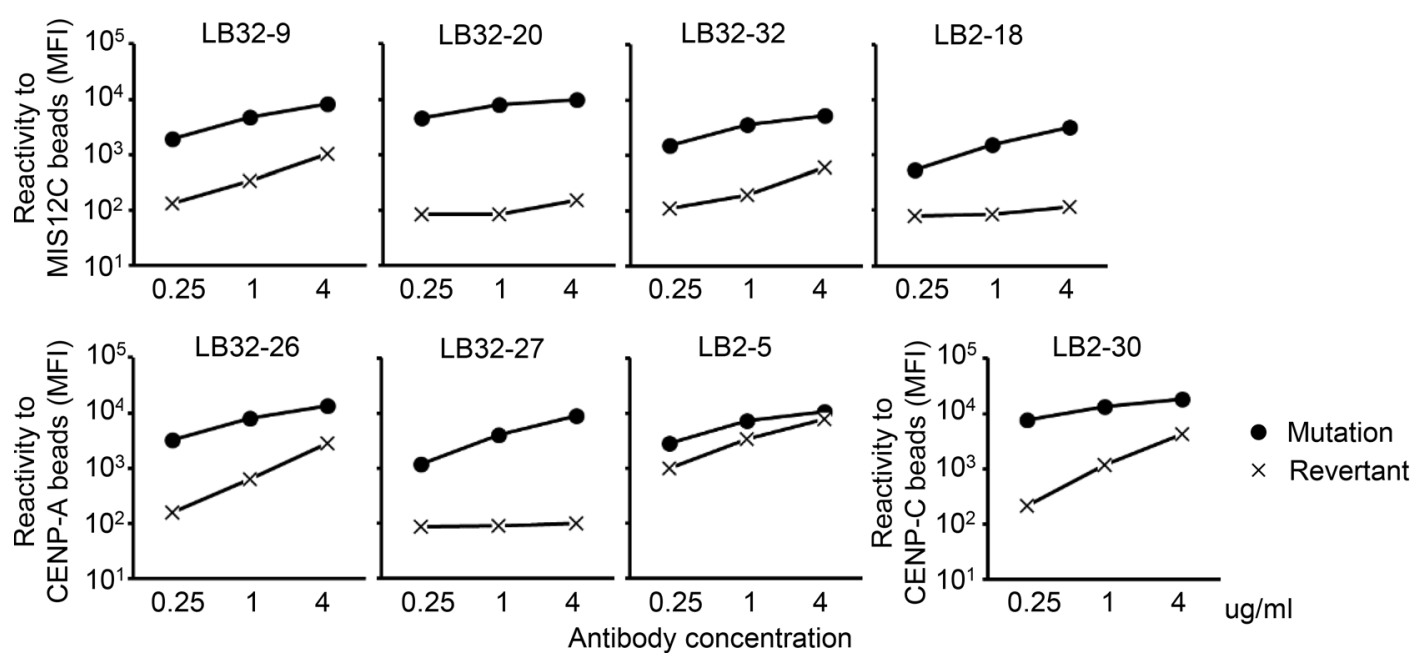

Figure 3 Direct evidence of the antigen-driven antibody selection of ACA. (A) GFP, MIS12 and PMF1, DSN1 and NSL1, or the whole MIS12C was coupled with streptavidin beads. The reactivity of anti-MIS12C antibody against each beads was measured by flow cytometry. (B) Anti-MIS12C, antiCENP-A and anti-CENP-C antibody and their revertants were tested by antigen-binding beads assay. The reactivity of antibody to each beads was measured by flow cytometry. ACA, anti-centromere antibody.

consist of two subcomplexes, MIS12-PMF1 and DSN1-NSL1 ${ }^{29}$; therefore, we coexpressed MIS12 and PMF1 or DSN1 and NSL1 and examined which MIS12C-specific antibody reacted to.

As shown in figure 3A, some of the anti-MIS12C antibodies recognised MIS12+PMF1, and others recognised DSN1+NSL1. Furthermore, there were antibodies that did not bind unless all four proteins were present. This result indicated that ACA recognised various sites of the target complex.

We also created revertant antibodies of ACA and examined their reactivity. As shown in figure $3 \mathrm{~B}$, all of the revertant antibodies showed decreased antigen reactivity. These results directly proved that ACA were selected in an antigen-driven manner in salivary glands.

\section{Serum ACA profile}

Next, we examined whether the antibody specific for each centromere-related protein could also be detected in serum. We recruited a large cohort consisting of 112 patients who had sicca symptoms and suspected of having SS; 35 patients with SSc; 10 patients with $\mathrm{PBC} ; 44$ patients who had two or more complications of SS, SSc and PBC; and 68 healthy controls (table 1), and the presence of antibody specific for CBX5, CENP-A, CENP-B, CENP-C and MIS12C was examined.

As shown in figure 4A, an IgG-type anti-MIS12C antibody as well as previously reported anti-CBX5, anti-CENP-A, antiCENP-B and anti-CENP-C antibody ${ }^{10}$ could be detected in serum, and the frequency of these antibody was highest in the overlap group. Because the IgA-type autoantibody was mainly produced in salivary glands, ${ }^{14}$ and because all cloned ACAs were IgA, we also profiled the prevalence of IgA-type autoantibody (figure 4B). Although IgA-type ACA was also detected in serum, the positivity was less clear than IgG-type ACA. Interestingly, the $\mathrm{IgG} / \operatorname{IgA}$ ratio for each antigen varied, and the highest $\operatorname{IgG} / \operatorname{IgA}$ ratio was observed for anti-CENP-B antibody (figure 4C).

\begin{tabular}{|c|c|c|c|c|c|}
\hline Disease group & HC & SS & SSc & PBC & Overlap \\
\hline & $n=68$ & $n=112$ & $n=35$ & $n=10$ & $n=44$ \\
\hline Age median (IQR) & $44(31-50)$ & $59(47-72)$ & $59(50-71)$ & $60(57-72)$ & $64(54-74)$ \\
\hline ACA positive $\%(n)$ & NA & $13(14)$ & $34(12)$ & $30(3)$ & $70(31)$ \\
\hline Anti-CENP-B antibody positive \% (n) & $1.5(1)$ & $13(14)$ & $37(13)$ & $30(3)$ & $73(32)$ \\
\hline
\end{tabular}

dcSSc, diffuse cutaneous SSc; IcSSc, limited cutaneous SSc; NA, not accessed; PBC, primary biliary cirrhosis; pSS, primary Sjögren's syndrome; SS, Sjögren's syndrome; sSS, secondary Sjögren's syndrome. 

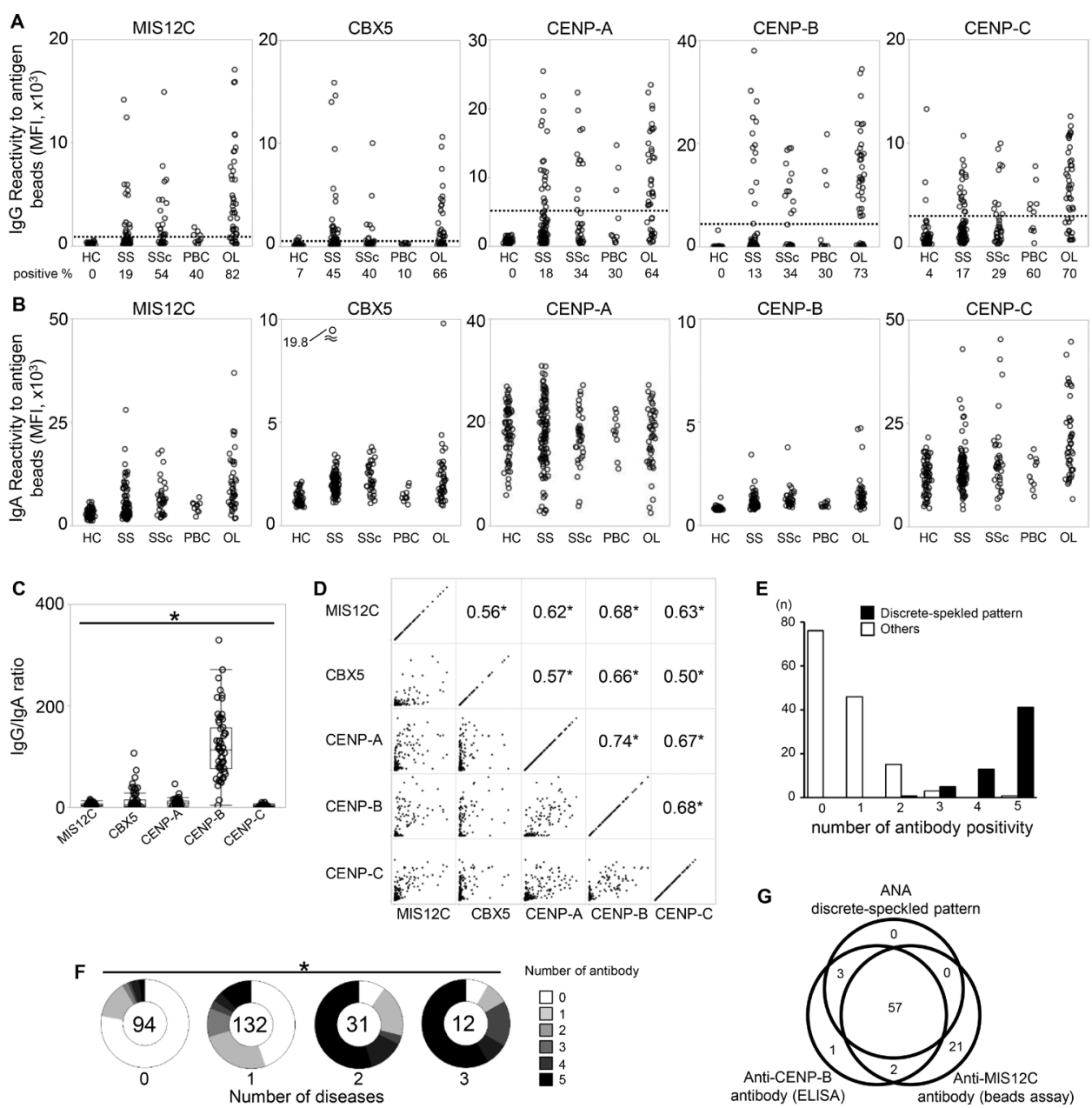

Figure 4 Serum profile of antibody against centromere-related proteins. Serum samples from HC ( $n=68)$, patients suspected of having SS ( $n=112)$, patients with SSC $(n=35), P B C(n=10)$ and OL diseases $(n=44)$ were analysed by antigen-binding beads assay. (A) serum IgG bound to autoantigenbinding beads was assessed by flow cytometry. The dotted line indicates a cut-off, which was determined by receiver operating characteristic (ROC) analysis. The prevalence of each antibody is presented under the group label. (B) Serum IgA bound to autoantigen-binding beads was assessed by flow cytometry. (C) The ratio of IgG/lgA in ACA-positive serum is shown. Wilcoxon's test. (D). The correlation between the serum IgG reactivity to each centromere-related antigen is shown. The lower left panels are two-dimensional plots of the MFI of antigen-binding beads assay, and the numbers in the upper right panels indicate the correlation coefficient calculated by Spearman's test. (E) The number of antibody that each patient had was counted. The patients whose serum showed a discrete-speckled pattern in an ANA test are shown in black bars, and the other patients are shown in white bars. (F) Patients were classified according to the number of diseases of SS, SSC and PBC, and counted how many ACA each patient had. $\chi^{2}$ test. The number of patients in each group are shown at the centre of each pie chart. (G) The overlap of serum ACA (discrete-speckled pattern by the ANA test), anti-CENP-B antibody detected by ELISA, and anti-MIS12C antibody detected by the beads assay is shown in a Venn diagram. ${ }^{*} \mathrm{p}<0.0001$. ACA, anti-centromere antibody; ANA, anti-nuclear antibody; $\mathrm{HCS}$, healthy controls; MFI, mean fluorescence intensity; OL, overlapping; PBC, primary biliary cirrhosis; SS, Sjögren's syndrome.

To investigate the relationships among these antibodies, we examined the correlations among the titres of these antibodies in each individual (figure 4D), and counted how many kinds of antibodies each patient had (figure 4E). We found that these antibodies were moderately to highly correlated with each other, and most of serum ACA-positive patients had antibodies to four or five antigens. This result indicated that serum ACA-positive patients have antibodies to various portions of the centromere 'complex' rather than antibodies to specific centromere proteins. The highest prevalence of ACA was observed in the overlap group (figure 4A). We further examined the relationship between the number of complicated diseases and the numbers of ACA, and found that the more these diseases the patients had, the more kinds of ACA became positive (figure 4F).

Next, we investigated the dissociation among ACA by ANA test, anti-CENP-B antibody by ELISA, and anti-MIS12C antibody by beads assay. As shown in figure 4G, many patients were commonly positive in all three assays; however, 21 patients were only anti-MIS12C antibody-positive. Although they may also include false positives, 6 of the 21 patients were also anti-CBX5 antibody-positive and 2 of the 21 patients were also anti-CENP-C antibody-positive, suggesting that in some patients, autoantibody could be detected only by the anti-MIS12C antibody test.

\section{Direct detection of ACA-secreting cells in salivary glands}

Finally, we directly confirmed the production of ACA in salivary glands. We expressed MIS12C, CBX5, CENP-A, CENP-B and CENP-C as GFP-fusion protein. Fresh-frozen sections of salivary glands were double stained with GFP-autoantigen and an antibody against CD138, the marker of ASCs. 


\begin{tabular}{|c|c|c|c|c|c|c|c|c|c|c|c|}
\hline Patient ID & S3 & S10 & Lip32 & Lip73 & Lip90 & S20 & Lip24 & Lip28 & Lip45 & Lip47 & Lip48 \\
\hline Sex & Female & Female & Female & Female & Female & Female & Female & Female & Female & Female & Female \\
\hline Age & 51 & 71 & 31 & 29 & 70 & 58 & 36 & 76 & 58 & 38 & 47 \\
\hline Disease & SSS, MCTD & pSS & pSS & sSS, SSC & pSS & pSS & pSS & pSS & non-SS & non-SS & non-SS \\
\hline ANA titre & $>1: 2560$ & $1: 640$ & $1: 640$ & $1: 2560$ & $1: 320$ & $1: 1280$ & $1: 640$ & $<1: 40$ & $<1: 40$ & $1: 40$ & $1: 80$ \\
\hline ANA type & $\mathrm{s}, \mathrm{C}$ & $h, d$ & $h, d, n$ & d & $h, c$ & s & s & & & $h, s$ & $h, s$ \\
\hline Serum anti-SSA52/60 antibody & + & + & - & - & - & + & + & - & - & - & - \\
\hline Serum anti-SSB antibody & + & - & - & - & - & + & + & - & - & - & - \\
\hline Serum anti-CENP-B antibody & + & + & + & + & + & - & - & - & - & - & - \\
\hline Serum rheumatoid factor & + & + & - & + & NA & + & + & - & NA & - & - \\
\hline Greenspan grade & 3 & 3 & 4 & 4 & 4 & 3 & 4 & 3 & 2 & 2 & 1 \\
\hline Medication & PSL $10 \mathrm{mg} /$ day & None & None & None & None & None & None & None & None & None & None \\
\hline \multicolumn{12}{|l|}{ Tissue ASCs } \\
\hline Anti-GFP antibody* & - & - & - & - & - & - & - & - & - & - & - \\
\hline Anti-MIS12C antibody* & +++ & +++ & +++ & +++ & +++ & - & - & - & - & - & - \\
\hline Anti-CBX5 antibody* & - & - & \pm & \pm & - & - & - & - & - & - & - \\
\hline Anti-CENP-A antibody* & \pm & \pm & + & ++ & \pm & - & \pm & \pm & - & - & - \\
\hline Anti-CENP-B antibody* & \pm & \pm & \pm & + & - & - & - & - & - & - & - \\
\hline Anti-CENP-C antibody* & +++ & +++ & ++ & +++ & ++ & - & - & - & - & - & - \\
\hline
\end{tabular}

*Slides were examined at a magnification of $\times 200 .-$, undetectable; \pm , one cell in multiple fields,,$+ 1-3$ cells in one field;,$++ 4-8$ cells in one field;,$+++>8$ cells in one field. c, cytosol; d, discrete-speckled; h, homogeneous; MCTD, mixed connective tissue disease; n, nucleolar; NA, not assessed; PSL, prednisolone; pSS, primary Sjögren's syndrome; $\mathrm{s}_{\text {, }}$ speckled; SS, Sjögren's syndrome; sSS, secondary Sjögren's syndrome.

The results are shown in table 2 , and representative images are shown in online supplementary figure 2. ACA-secreting cells were detected in salivary glands of ACA-positive patients. We previously showed the patient who did not have serum ACA but did have ACA-secreting cells in salivary glands; however, patients like that one may not be frequent.

Among ACA-related antigens, anti-MIS12C ASCs were detected most frequently, followed by anti-CENP-C ASCs. There were few antibody to other antigens, especially CBX5 and CENP-B. Interestingly, this frequency was somewhat consistent with the frequency of cloned ACA (11 anti-MIS12C antibodies, 10 anti-CENP-C antibodies, 2 anti-CBX antibodies, 1 anti-CENP-A antibody, and no anti-CENP-B antibody among 24 ACAs). As shown in supplementary figure S2, anti-MIS12C antibody, anti-CENP-A antibody, and anti-CENP-C antibody were detected in a same area of serial sections, indicating that these antibodies were produced in a centromere 'complex'-driven manner.

\section{DISCUSSION}

In this study, we efficiently and comprehensively cloned antibodies from human salivary glands and investigated their reactivity. We succeeded in discovering direct evidence that ACA in addition to anti-SSA/SSB antibody are produced in lesions of SS in an antigen-driven manner. Moreover, we discovered a new autoantigen, MIS12C, in this process. Finally, we profiled the prevalence of ACA against several corresponding antigens in patients with SS, SSc, and PBC, and found that ACA-positive patients have antibodies against various sites of centromere 'complex' regardless of disease.

One of the advantages of our study is that incorporating recent single-cell techniques ${ }^{32}$ enabled the efficient cloning of immunoglobulin sequences, resulting in successful cloning with $73 \%$ of sorted cells. Because ASCs were sorted without selection by their isotype, we could reproduce humoral immune responses in salivary glands in vitro without bias. This was confirmed by similarity between the antigen profiles of cloned ACA and ASCs in salivary gland tissues.

Another advantage of our study is the development of antigenbinding beads assay, which enable to detect autoantibodies recognising conformational epitopes. We found many anti-SSA52 and SSB antibodies that were negative by ELISA but positive by beads assay, but not vice versa. The novel target of ACA, MIS12C, was also found using this assay. These results suggest that most of autoantibodies target against conformational molecules, and a part of these antibodies recognise the linear parts of conformational molecules, which can detect by ELISA. This hypothesis enables to explain the discrepancy between previous studies and our results. In our study, most patients had several kinds of ACAs simultaneously, whereas the corresponding antigens of ACA were reported to differ depending on the disease ${ }^{1011}$; our beads assay used recombinant protein produced in mammalian cell line, whereas previous studies used fragmented protein expressed by Escherichia coli or protein generated by in vitro transcription/translation.

We showed that ACA-positive patients have antibodies against various sites of centromere complex regardless of disease. The relationship between ACA and corresponding diseases is similar to the concept of anti-ARS antibody syndrome, in which anti-ARS antibody-positive patients often develop one or more diseases involving myositis, interstitial pneumonia and skin symptoms, ${ }^{33} 34$ or mixed connective tissue disease (MCTD), ${ }^{35}$ in which anti-U1RNP antibody-positive patients often develop one or more phenotypes resembling systemic lupus erythematosus (SLE), SSc, and myositis. Therefore, these ACA-positive patients may be classified as 'ACA-related disease' in addition to classical disease category. Additional study is desired for classification of these diseases.

We identified three polyreactive antibodies; two of them recognised disease-specific autoantigens such as SSB, while they bound to a specific part of the antigen, and reactivity decreased after SHM reversion. These results indicated that polyreactivity 
may accidentally emerge at least in part as a result of selection against a specific antigen.

One group reported that approximately one-third of peripheral naïve $B$ cells from patients with SS recognises SSA52, ${ }^{22}$ whereas we cloned only seven anti-SSA52 antibodies. In our antibody panel, some antibodies could bind to SSA60 and/or SSB and SSA52 simultaneously, suggesting the possibility that SSA52specific antibody actually occurs less frequently, and most of so far 'anti-SSA52 antibody' are rather non-specific.

Anti-MIS12C antibody and anti-CENP-C antibody were mainly found among the cloned antibodies and by tissue staining, whereas few anti-CENP-B antibodies were found. The isotype of all of the cloned ACA were IgA, and serum IgG/IgA ratio of antiMIS12C and anti-CENP-C antibody were also low. In contrast, serum IgG/IgA ratio of anti-CENP-B antibody was much higher. This finding indicated the possibility that antibody-producing mechanisms may differ depending on the corresponding antigens. For example, anti-MIS12C antibody and anti-CENP-C antibody were produced against centromere complex in salivary glands, whereas anti-CENP-B antibody was rarely produced in salivary glands for some reasons (eg, antigen is not exposed on the surface of complex, or antigenicity is low in the conformation of complex), but they are produced in other places, such as lymph nodes, after undergoing antigen processing.

In this study, we directly showed answers for some of the scientific questions about autoantibody, that anti-SSA/SSB antibody and ACA were produced in local lesions of SS in an antigen-driven manner, and that autoantibody can be produced against not only individual proteins but also protein complexes. Our strategy, separating various antibody included in serum into monoclonal populations, is somewhat similar to the relationship between bulk and single-cell RNA sequencing, and could produce the information with higher resolution. Because anti-citrullinated protein antibody-secreting cells have been detected in synovium of patients with rheumatoid arthritis, ${ }^{36}$ it seems common that disease-specific antibody are produced locally in lesions. However, there are many remaining questions about autoantibody, such as why SSA/SSB and the centromere 'complex' can be targets in salivary glands, and whether these antibodies are involved in the destruction of salivary glands. In addition, new hypotheses were created from this study, including the relationship between autoantibodies and disease classification, and the difference in reactivity between serum and local antibodies. Further studies are needed to resolve these questions.

Acknowledgements We thank Harumi Kondo and Mayumi Ota for collecting clinical samples. This study was supported by the Collaborative Research Resources, School of Medicine, Keio University for technical assistances. pEFs vector was kindly gifted from Dr A. Yamashita, Yokohama City University School of Medicine, Japan.

Contributors Study design: MT, KS, KT and TT. Data acquisition: MT, YK, HY, KI, HS, KT and HA. Data analysis and interpretation: MT and KS. Manuscript drafting: MT, KS and TT. All authors approved the final version of the manuscript.

Funding This work was supported by JSPS KAKENHI Grant Number JP 16K19609 and JP 17 H04216.

Competing interests MT, KS and TT have applied for a patent of anti-MIS12C antibody as diagnostic marker. The remaining authors declare no competing interests.

Patient consent for publication Written informed consent was obtained from all participating individuals.

Ethics approval Ethics approval was obtained from the Institutional Review Board of Keio University School of Medicine.

Provenance and peer review Not commissioned; externally peer reviewed.

Data availability statement All data relevant to the study are included in the article or uploaded as supplementary information.

Open access This is an open access article distributed in accordance with the Creative Commons Attribution Non Commercial (CC BY-NC 4.0) license, which permits others to distribute, remix, adapt, build upon this work non-commercially, and license their derivative works on different terms, provided the original work is properly cited, appropriate credit is given, any changes made indicated, and the use is non-commercial. See: http://creativecommons.org/licenses/by-nc/4.0/.

\section{ORCID iD}

Masaru Takeshita http://orcid.org/0000-0002-3281-6595

\section{REFERENCES}

1 Didier K, Bolko L, Giusti D, et al. Autoantibodies associated with connective tissue diseases: what meaning for clinicians? Front Immunol 2018;9:541.

2 Elkon K, Casali P. Nature and functions of autoantibodies. Nat Clin Pract Rheumatol 2008:4:491-8

3 Nocturne G, Mariette X. B cells in the pathogenesis of primary Sjögren syndrome. Nat Rev Rheumatol 2018;14:133-45.

4 Shiboski CH, Shiboski SC, Seror R, et al. American College of Rheumatology/European League against rheumatism classification criteria for primary Sjogren's syndrome: a consensus and data-driven methodology involving three international patient cohorts. Ann Rheum Dis 2016;2017:9-16.

5 Routsias JG, Tzioufas AG. Sjögren's Syndrome-Study of Autoantigens and Autoantibodies. Clin Rev Allergy Immunol 2007;32:238-51.

6 Tan EM, Rodnan GP, Garcia I, et al. Diversity of antinuclear antibodies in progressive systemic sclerosis. anti-centromere antibody and its relationship to crest syndrome. Arthritis Rheum 1980;23:617-25.

7 Parveen S, Morshed SA, Nishioka M. High prevalence of antibodies to recombinant CENP-B in primary biliary cirrhosis: nuclear immunofluorescence patterns and ELISA reactivities. J Gastroenterol Hepatol 1995;10:438-45.

8 van den Hoogen F, Khanna D, Fransen J, et al. Classification criteria for systemic sclerosis: an American College of rheumatology/European League against rheumatism collaborative initiative. Ann Rheum Dis 2013;2013:1747-55.

9 Rothfield N, Whitaker D, Bordwell B, et al. Detection of anticentromere antibodies using cloned autoantigen cenp-Bl. Arthritis \& Rheumatism 1987;30:1416-9.

10 Tanaka N, Muro Y, Suzuki Y, et al. Anticentromere antibody-positive primary Sjögren's syndrome: epitope analysis of a subset of anticentromere antibody-positive patients. Modern Rheumatology 2017;27:115-21.

11 Gelber ACet al. Distinct recognition of antibodies to centromere proteins in primary Sjogren's syndrome compared with limited scleroderma. Ann Rheum Dis 2006;65:1028-32.

12 Tsukamoto M, Suzuki K, Takeuchi T. Clinical and Immunological Features of Anticentromere Antibody-Positive Primary Sjögren's Syndrome. Rheumatology and Therapy 2018;5:499-505.

13 Katano K, Kawano M, Koni I, et al. Clinical and laboratory features of anticentromere antibody positive primary Sjogren's syndrome. The Journal of rheumatology 2001;28:2238-44

14 Horsfall AC, Rose LM, Maini RN. Autoantibody synthesis in salivary glands of Sjögren's syndrome patients. J Autoimmun 1989;2:559-68.

15 Tengnér $\mathrm{P}$, Halse AK, Haga HJ, et al. Detection of anti-Ro/SSA and anti-La/SSB autoantibody-producing cells in salivary glands from patients with Sjögren's syndrome. Arthritis Rheum 1998;41:2238-48.

16 Salomonsson S, Jonsson MV, Skarstein K, et al. Cellular basis of ectopic germinal center formation and autoantibody production in the target organ of patients with Sjögren's syndrome. Arthritis \& Rheumatism 2003:48:3187-201.

17 Gellrich S, Rutz S, Borkowski A, et al. Analysis of V(H)-D-J(H) gene transcripts in B cells infiltrating the salivary glands and lymph node tissues of patients with Sjögren's syndrome. Arthritis Rheum 1999;42:240-7.

18 Dörner Tet al. Immunglobulin repertoire analysis provides new insights into the immunopathogenesis of Sjögren's syndrome. Autoimmun Rev 2002;1:119-24.

19 Stott DI, Hiepe F, Hummel M, et al. Antigen-Driven clonal proliferation of B cells within the target tissue of an autoimmune disease. the salivary glands of patients with Sjögren's syndrome. J Clin Invest 1998;102:938-46.

20 Tiller T, Meffre E, Yurasov S, et al. Efficient generation of monoclonal antibodies from single human B cells by single cell RT-PCR and expression vector cloning. J Immunol Methods 2008;329:112-24.

21 Reddy J, Corsiero E, Sutcliffe N, et al. Accumulation of Self-Reactive Naïve and Memory B Cell Reveals Sequential Defects in B Cell Tolerance Checkpoints in Sjögren's Syndrome. PLOS ONE 2014;9:e114575.

22 Glauzy S, Sng J, Bannock JM, et al. Defective early B cell tolerance checkpoints in Sjögren's syndrome patients. Arthritis Rheumatol 2017;69:2203-8.

23 Glauzy S, Boccitto M, Bannock JM, et al. Accumulation of Antigen-Driven Lymphoproliferations in Complement Receptor 2/CD21 -/low B Cells From Patients With Sjögren's Syndrome. Arthritis Rheumatol 2018;70:298-307.

24 Maier-Moore JS, Koelsch KA, Smith K, et al. Antibody-Secreting cell specificity in labial salivary glands reflects the clinical presentation and serology in patients with Sjögren's syndrome. Arthritis Rheumatol 2014;66:3445-56.

25 Mietzner B, Tsuiji M, Scheid J, et al. Autoreactive IgG memory antibodies in patients with systemic lupus erythematosus arise from nonreactive and polyreactive precursors Proc Natl Acad Sci U SA 2008;105:9727-32. 
26 James LC, Keeble AH, Khan Z, et al. Structural basis for PRYSPRY-mediated tripartite motif (TRIM) protein function. Proc Natl Acad Sci U S A 2007;104:6200-5.

27 Burbelo PD, Teos LY, Herche JL, et al. Autoantibodies against the immunoglobulinbinding region of Ro52 link its Autoantigenicity with pathogen neutralization. Sci Rep 2018;8:3345.

28 Scofield RH, Farris AD, Horsfall AC, et al. Fine specificity of the autoimmune response to the Ro/SSA and La/SSB ribonucleoproteins. Arthritis \& Rheumatism 1999;42:199-209.

29 Petrovic A, Keller J, Liu Y, et al. Structure of the Mis12 complex and molecular basis of its interaction with CENP-C at human kinetochores. Cell 2016;167:e1015:1028-40.

30 Obuse C, Iwasaki 0, Kiyomitsu T, et al. A conserved Mis 12 centromere complex is linked to heterochromatic HP1 and outer kinetochore protein Zwint-1. Nat Cell Biol 2004:6:1135-41.

31 Fachinetti D, Han JS, McMahon MA, et al. Dna sequence-specific binding of CENP-B enhances the fidelity of human centromere function. Dev Cell 2015;33:314-27.
32 Picelli S, Faridani OR, Björklund AK, et al. Full-Length RNA-seq from single cells using Smart-seq2. Nat Protoc 2014;9:171-81.

33 Lega J-C, Fabien N, Reynaud Q, et al. The clinical phenotype associated with myositisspecific and associated autoantibodies: a meta-analysis revisiting the so-called antisynthetase syndrome. Autoimmun Rev 2014;13:883-91.

34 Love LA, Leff RL, Fraser DD, et al. A new approach to the classification of idiopathic inflammatory myopathy: myositis-specific autoantibodies define useful homogeneous patient groups. Medicine 1991;70:360-74.

35 Tani C, Carli L, Vagnani S, et al. The diagnosis and classification of mixed connective tissue disease. J Autoimmun 2014;48-49:46-9.

36 Steen J, Forsström B, Sahlström P, et al. Recognition of amino acid motifs, rather than specific proteins, by human plasma cell-derived monoclonal antibodies to posttranslationally modified proteins in rheumatoid arthritis. Arthritis Rheumatol 2019;71:196-209. 